\title{
IMPACT OF CORRELATION AND COUPLING ON THE CAPACITY OF MIMO SYSTEMS
}

\author{
Nicolae Chiurtu, Bixio Rimoldi and \\ Emre Telatar
Swiss Federal Institute of Technology, Lausanne, Switzerland
e-mail: \{nicolae.chiurtu, bixio.rimoldi, emre.telatar\}@epfl.ch

\begin{abstract}
In this work we consider multiple antenna systems in which a large number of antennas occupy a given physical volume and we investigate the behavior of the capacity when increasing the number of antennas. In this regime the assumptions of the standard multiple antenna models become questionable. We introduce several new channel models that better fit this scenario and show that for such "spatially dense" multiple antenna systems one should expect the behavior of the capacity to be qualitatively different than what the standard multiple antenna models predict.
\end{abstract}

\section{INTRODUCTION}

It is widely recognized that the use of multiple antennas at both ends of a communication system can greatly increase the capacity of the link. In particular, if the entries of the channel transfer matrix are assumed to be i.i.d. Gaussian random variables and known at the receiver, the capacity increases linearly with the minimum number of transmit and receive antennas [1]. With the use of multiple antennas, the space becomes a new resource to be exploited towards increasing the capacity of the communication systems. However, in practical applications, the space allocated to the transmitter and the receiver is in general limited, and we have a fixed volume to place the transmit/receive antennas. It is clear that if we start packing many antennas in a limited volume they will begin to couple and the capacity gains predicted in [1] will diminish considerably. This suggests that there should be a limit on the capacity that can be achieved using a given volume.

Motivated by these considerations, we investigate the case when more and more antennas are packed into a given physical volume. For alternative approaches to the question of physical limitation of space, see [2], [3]. In the next section we present our first proposition of the channel model for such systems.

\footnotetext{
${ }^{1}$ This work was partially supported by Nokia.
}

\author{
Volker Pauli \\ Institute for Electro Engineering \\ Electronics und Information Technology \\ Friedrich-Alexander-Universität \\ Erlangen-Nürnberg, Germany \\ e-mail: volker_pauli@web.de
}

\section{CHANNEL MODEL AND RESULTS}

We consider a system with $t$ transmit antennas and $r$ receive antennas in which the received vector $\mathbf{v} \in \mathbb{C}^{r}$ depends on the transmitted vector $\mathbf{u} \in \mathbb{C}^{t}$ via

$$
\mathbf{v}=H \mathbf{u}+\mathbf{w}
$$

where $H \in \mathbb{C}^{r \times t}$ is the channel transfer matrix and $\mathbf{w}$ is zero-mean complex circular symmetric Gaussian noise. We assume that $E\left[\mathbf{w w}^{\dagger}\right]=\sigma^{2} I_{r}$. The transmitter is constrained in its total power, i.e., $\mathcal{E}\left[\mathbf{u}^{\dagger} \mathbf{u}\right] \leq P$ or equivalently $\operatorname{tr}(Q) \leq$ $P$ where $Q=\mathcal{E}\left[\mathbf{u u}^{\dagger}\right]$ is the input covariance matrix.

In most physical scenarios, the gain $H_{j i}$ from the $i^{\text {th }}$ transmitter to the $j^{\text {th }}$ receiver is due to the agglomeration of many small contributions and thus it is reasonable to assume that the collection $\left\{H_{j i}\right\}$ is jointly Gaussian, and circularly symmetric. Furthermore, it is also common to assume that the $\left\{H_{j i}\right\}$ form an independent collection.

While this last assumption can be justified in cases for which the transmitting antennas are separated from each other by some multiple (e.g., 1/4) of the wavelength and likewise for the recejving antennas, when we wish to talk about a large number of antennas packed into a fixed volume this assumption becomes questionable. Furthermore, if one fixes the variances of $H_{j i}$ and holds the total transmitted power constant, the total signal power received by the receiving antennas scales with $r$. If the receiving antennas are assumed to occupy a given volume, to assume that one can increase the total received power by placing more receiving antennas into this volume sounds very dubious. The concerns raised above leads us to consider a multiple antenna system with $r$ receiving and $t$ transmitting antennas where

$$
\mathbf{v}=r^{-1 / 2} H \mathbf{u}+\mathbf{w}
$$

The scaling of the information carrying component $H \mathbf{u}$ of the received signal by $r^{-1 / 2}$ ensures that the total received power remains bounded even when we increase $r$. We will assume that the entries of the channel gain matrix $H$ are 
jointly circularly symmetric and Gaussian, and have a correlation structure given by

$$
\mathcal{E}\left[H_{j i} H_{l k}^{*}\right]=C_{k i} D_{j l} .
$$

One justification for the above form is the following. Imagine that the signals sent from the transmitted antennas first travel to a 'cloud' of scatterers, and then from this cloud they travel to the receiving antennas. Assume that once scattered by the cloud, the signals have lost any statistical "information" about their origins. This translates to the assumption that $H_{j i}=A_{i} B_{j}$ where $A_{i}$ is the gain from the transmitter $i$ to the cloud, $B_{j}$ the gain from the cloud to the receiver $j$ and that the ' $A$ ' gains and ' $B$ ' gains are independent. However, it is likely that when antennas $i$ and $k$ are close to each other $A_{i}$ and $A_{k}$ will be correlated, and similarly for $B_{j}$ and $B_{l}$. If we let these correlations be $C_{k i}$ and $D_{j l}$ we obtain (3). Since $C$ and $D$ represent correlation matrices, it becomes clear that we should assume that $C$ and $D$ are non-negative definite matrices. Note that the same correlation structure is assumed in [5], [6].

For the purposes of this paper, assume (quite optimistically) that the receiver is aware of the realization of $H$, but that the transmitter only knows the statistics of $H$, namely, $C$ and $D$. From [1] we know that in our settings, the capacity is given by

$$
C=\max _{Q: \operatorname{tr}(Q) \leq P} \mathcal{E} \log \operatorname{det}\left(I_{r}+\frac{1}{r} H Q H^{\dagger}\right) .
$$

After some algebra, we find that for a channel correlation matrix as in equation (3) the capacity is

$$
C=\max _{\tilde{Q}: \operatorname{tr}(\tilde{Q}) \leq P} \mathcal{E} \log \operatorname{det}\left(I_{r}+\frac{1}{r} G \widetilde{Q} G^{\dagger}\right),
$$

where $G=\Lambda^{1 / 2} W M^{1 / 2}, W$ contains proper Gaussian $\mathcal{N}(0,1)$ random variables, $\Lambda$ and $M$ contain the singular values of $D$ and $C$ respectively and $\widetilde{Q}$ is a unitary transformation of $Q$.

Let us now bring in the path gain correlations due to the proximity of the receiving antennas to each other. Consider a fixed volume of space into which we pack more and more receiving antennas. In this case, we can label the antennas such that $(j-1) / r$ indicates the physical location of antenna $j$ when $r$ receiving antennas are present. It now makes sense to assume that the correlation between the $j^{\text {th }}$ and $l^{\text {th }}$ receiving antennas is a function of their physical positions only, thus, $D_{j l}$ is a function of $(j-1) / r$ and $(l-1) / r$, i.e., $D_{j l}=d((j-1) / r,(l-1) / r)$. Since $d:[0,1]^{2} \rightarrow \mathbb{C}$ models a covariance function, it is Hermitian and positive definite, i.e., $d(\alpha, \beta)=d(\beta, \alpha)^{*}, \iint g(\alpha)^{*} d(\alpha, \beta) g(\beta) d \alpha d \beta \geq 0$ for any $g$. In addition, let us assume that $d$ is continuous and that

$$
\iint|d(\alpha, \beta)|^{2} d \alpha d \beta<\infty
$$

We will say that such a system is spatially dense at the receiver. For the time being, let the transmitting antennas be 'sparse', i.e., suppose that $C=I_{t}$.

With these assumptions, one can show that the eigenvalues of the matrix $D / r$ for large $r$ approach the (point) spectrum of the operator $d$ : Notice first that the operator $d$ only has a point spectrum (see, e.g. [7]) and that the eigenvalues of $D / r$ are exactly those of the operator $d_{r}:[0,1]^{2} \rightarrow \mathbb{C}$, where

$$
d_{r}(\alpha, \beta)=d(\lfloor r \alpha\rfloor / r,\lfloor r \beta\rfloor / r) .
$$

Second, note that for any $L_{2}[0,1]$ function $g$,

$\iint g(\alpha)^{*} d_{r}(\alpha, \beta) g(\beta) d \alpha d \beta \rightarrow \iint g(\alpha)^{*} d(\alpha, \beta) g(\beta) d \alpha d \beta$

with increasing $r$. Combining this with the extremal representation of the eigenvalues of Hermitian operators and matrices we see that the eigenvalues of $D / r$ approach the spectrum of $d$.

It is now easy to see that the capacity of this system in the limit of large $r$ and $t$ approaches a finite limit. If one assumes that the transmitting antennas are also spatially dense, then a more involved argument leads one to conclude that the effect of large $r$ and $t$ on the capacity is to scale the power by a factor $t$. In other words, if $C(r, t, P)$ denotes the capacity for a given number of receiving and transmitting antennas and available power $P, C(r, t, P)$ behaves like $F(t P)$ for large $r$ and $t$ for a suitable function $F$. This could to be due to the feasibility in the model of the beam-forming at the transmitter when there is a large amount of correlation between the gains from transmitting antennas to the scattering medium. However, from the analysis performed so far we are not able to give a reasonable physical explanation of this artificial effect, which seems to be due to a flaw in our channel model. Therefore, in the following we are going to review the channel model and consider in our analysis the mutual coupling between antennas. This effect is due to the electromagnetic interactions between antennas and has been studied extensively in the past by antenna arrays designers. There are several possibilities to consider mutual coupling between antennas. One approach within our framework is to consider the $Z$-Matrix of the system.

\section{Z-PARAMETER APPROACH}

To include mutual coupling between antennas in our model, we take a very general approach. We will consider the entire system as one large multi-port where each antenna is associated with a port. In this model in principle all ports are coupled. We will determine the channel transfer function in terms of the $Z$-parameters.

Figure 1 depicts this multi-port representation of our channel. For clarity we arranged the ports in such a way that those associated with transmit antennas are on the right 


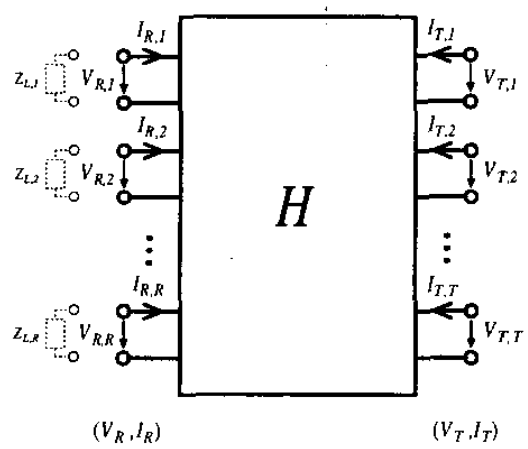

Fig. 1. Multi-port representation

and those related to the receive antennas are set on the left. We denote by $V_{T}$ the column vector of the voltages at the transmitter, that is, $V_{T}=\left(V_{T, 1}, V_{T, 2}, \ldots, V_{T, t}\right)^{T}$. The vectors $V_{R}, I_{R}$ and $I_{T}$ are defined similarly.

Our multi-port can be described through $Z$-parameters as follows:

$$
\left(\begin{array}{c}
V_{R} \\
V_{T}
\end{array}\right)=\left(\begin{array}{ll}
Z_{R R} & Z_{R T} \\
Z_{T R} & Z_{T T}
\end{array}\right) \cdot\left(\begin{array}{c}
I_{R} \\
I_{T}
\end{array}\right) .
$$

The sub-block matrices on the main diagonal $Z_{R R}$ and $Z_{T T}$ characterize the mutual coupling within the receive and transmit arrays, respectively. $Z_{R T}$ stands for the "transmission impedance" from the transmit array to the receive array. Similarly, $Z_{T R}$ stands for the transmission impedance from the receive array to the transmit array.

We assume that we fix a certain voltage $V_{T}$ at the transmitter, and at the receiver we put loads (see Figure 1). We denote the diagonal matrix of the loads at the receiver by $Z_{L}$, i.e., $Z_{L}=\operatorname{diag}\left(Z_{L, 1}, Z_{L, 2}, \ldots, Z_{L, r}\right)$. Under these circumstances the currents and voltages at the receiver are related through the loads $V_{R}=-Z_{L} I_{R}$. Plugging this into equation (6) we can easily find $V_{R}=f\left(V_{T}\right)$ :

$V_{R}=\left(I_{r}+Z_{R R} Z_{L}^{-1}-Z_{R T} Z_{T T}^{-1} Z_{T R} Z_{L}^{-1}\right)^{-1} Z_{R T} Z_{T T}^{-1} V_{T}$,

where $I_{r}$ denotes the $r$-dimensional identity matrix.

We can see that the above expression gives us the (voltage) transfer matrix in terms of the two transmission matrices $Z_{R T}, Z_{T R}$ and the two coupling matrices $Z_{R R}$ and $Z_{T T}$ at the receiver and transmitter, respectively.

The entries of the matrices $Z_{T T}, Z_{R T}, Z_{T R}$ and $Z_{R R}$ are related to the distance between the corresponding antennas. Since the distance between transmitter and receiver is generally much larger than the distance between elements of an array, the entries of the coupling matrices $Z_{T T}$ and $Z_{R R}$ are much larger than those of the transmission matrices $Z_{R T}$ and $Z_{T R}$. Since the last term inside the brackets contains the product of $Z_{R T}$ and $Z_{T R}$ it is reasonable to neglect it, thereby obtaining

$$
\begin{aligned}
V_{R} & =Z_{L}\left(Z_{L}+Z_{R R}\right)^{-1} Z_{R T} Z_{T T}^{-1} V_{T} \\
& =Z_{L}\left(Z_{L}+Z_{R R}\right)^{-1} Z_{R T} I_{T} .
\end{aligned}
$$

The term $Z_{R T} I_{T}$ represents the voltages at the receive antennas induced by the currents $I_{T}$ at the transmit antennas, when the coupling between the receive antennas is not yet accounted for (e.g. see [4]). We denote these voltages by

$$
V_{R T}=Z_{R T} I_{T}
$$

The voltages $V_{R T}$ are called "open-circuit voltages", as they represent those parts of the terminal voltages that are caused only by the incident electromagnetic field when all the antennas are open-circuited. Combining the previous three equations we obtain

$$
V_{R}=V_{R T}+Z_{R R} I_{R}
$$

which tells us that the terminal voltage at a receive antenna is a superposition of a voltage induces by an incoming electromagnetic field and voltages induced via coupling due to currents on the antennas of the array. Thus, we obtain a very simple and intuitive channel model which agrees with the models considered by antenna array designers [4].

In the following we are going to review the input power constraint. In our channel model we consider the voltages $V_{T}$ applied to the terminals of the antennas to be the input signals of the channel. Remember that the channel (voltage) transfer matrix was found to be

$$
H=Z_{L}\left(Z_{L}+Z_{R R}\right)^{-1} Z_{R T} Z_{T T}^{-1}
$$

So far, the power constraint in the determination of capacity was given by

$$
\operatorname{tr}\left(\mathbf{u u ^ { \dagger }}\right) \leq P,
$$

where $\mathbf{u}$ was the vector of the input signal and $P$ denoted the maximal total real power available.

As we have seen in the previous equations, when we have coupling between antennas the terminal impedances of the transmit antennas will change depending on the distance between antennas. Then, the relation between voltages and currents at the transmit array will change as well. On the other hand, we see that the power constraint in (11) depends only on the input voltages. Thus, it can happen that the real power consumed (which depends on both currents and voltages) may increase even if the power constraint in (11) is fulfilled. A more realistic power constraint is

$$
\operatorname{Re}\left\{I_{T}^{\dagger} V_{T}\right\}=\operatorname{Re}\left\{I_{T}^{\dagger} Z_{T T} I_{T}\right\} \leq P .
$$

Here $P$ is the total real power supplied to the array. After some simple algebra we obtain the new power constraint as

$$
\operatorname{tr}(Y Q) \leq P .
$$


where $Y=\frac{Z_{x I}^{-\dagger}+Z_{I x}^{-1}}{2}$ and $Q=V_{T} V_{T}^{\dagger}$. With the new power constraint, the capacity formula becomes:

$$
C=\max _{Q: \operatorname{tr}\left(\sqrt{Y} Q \sqrt{Y^{\dagger}}\right) \leq P} \mathcal{E}\left\{\log \left(\operatorname{det}\left(I_{r}+H Q H^{\dagger}\right)\right)\right\},
$$

with the voltage transfer matrix $H$ from equation (7).

In order to simplify the power constraint, we define $\widehat{Q}=$ $\sqrt{Y} Q \sqrt{Y}^{\dagger}$ and the capacity expression becomes

$$
C=\max _{\widehat{Q}: \operatorname{tr}(\hat{Q}) \leq \dot{P}} \mathcal{E}\left\{\log \left(\operatorname{det}\left(I_{r}+\hat{H} \widehat{Q} \hat{H}^{\dagger}\right)\right)\right\} .
$$

where $\widehat{H}=H \sqrt{Y}^{-1}$.

Since the optimization over the covariance matrix $\widehat{Q}$ is difficult to do analytically, we performed Matlab simulations where we computed $\widehat{Q}$ numerically. We have considered linear arrays where the antennas are spaced uniformly in the corresponding lengths of the arrays $d_{T}, d_{R}$. Once chosen, $d_{T}$ and $d_{R}$ are kept constant while we increase the number of the antennas. As a basis for the new channel model we use the correlation channel introduced in Section 1. In other words, the transfer matrix $Z_{R T}$ has the same correlation structure as described in equation (3). In order to show more clearly the effects of the mutual coupling, we will also include in our plots the capacity curves corresponding to the channel model from Section 1. The coupling between antennas was computed with the formulas corresponding to thin linear antennas of length $\lambda / 2$.

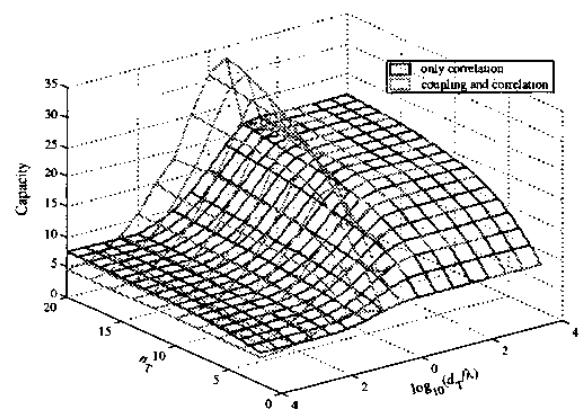

Fig. 2. Capacity as a function of $t$ and $d_{T}$ for $r=10$ and $d_{R}=1000 \lambda$

Figure 2 shows capacity in the case when the antennas at the receiver are fixed (both as number and position). The number of receive antennas is 10 and the length of the entire receive array is $1000 \lambda$ so that the assumption of no correlation at receiver side is valid. We observe that in the region where the transmit antennas are sparse the two curves coincide as expected since in that region we have no coupling and no correlation. On the other extreme, when the transmit antennas are very close by, coupling and correlation are both present. As expected (see analysis in Section 1) the capacity computed only with correlation is increasing with the number of transmit antennas. However, when we have both correlation and coupling the capacity saturates with the increasing number of transmit antennas. This shows that the presence of the coupling in the channel model eliminates the artificial effects observed in Section 1. In the region of interelement spacing of about $0.25 \lambda$ we found that despite some correlation in the channel, there is actually a major gain in the capacity. This seems to agree with G.H. Brown's antenna theory which was confirmed in practice by J.D. Kraus' W8JK antennas [4]. This theory predicts that for arrays with antennas spaced at distances about $0.25 \lambda$ there can be actually be a gain in the field strength. This effect seems to be due to the coupling between the antennas which leads to a decorrelation of the signals.

\section{CONCLUSION}

Spatially dense multiple antenna systems exhibit qualitatively different behavior from their sparse counterparts in the limit of large number of antennas. This paper attempts to point out the gross differences between such systems by computing the capacity associated with several new proposed channel models which are more appropriate to the case when many antennas are packed into a limited volume.

\section{REFERENCES}

[1] I. E. Telatar. Capacity of multi-antenna Gaussian channels. European Transactions On Telecommunications, vol. 10, pp. 585-95, November/December 1999.

[2] T. L. Marzetta. Fundamental limitations on the capacity of wireless links that use polarimetric antenna arrays. Proceedings of the IEEE International Symposium on Information Theory, 2002.

[3] J. W. Wallace and M. A. Jensen. Intrinsic Capacity of the Continuous-Space Electromagnetic Channel. Submitted to IEEE Trans. on Information Theory.

[4] J. D. Kraus. Antennas. Second Edition, McGraw-Hill International, 1988.

[5] A. M. Sengupta and P. P. Mitra. Capacity of multivariate channels with multiplicative noise: I. Random matrix techniques and large- $\mathrm{N}$ expansions for full transfer matrices. Bell Labs, Technical Report, May 1999.

[6] D.-S. Shiu, G. J. Foschini, M. J. Gans and J. M. Kahn. Fading Correlation and Its Effect on the Capacity of Multielement Antenna Systems. IEEE Trans. on Comm., vol 48, No. 3, March 2000.

[7] F. Riesz and B. Sz.-Nagy, Functional Analysis. New York, Dover Publications, 1990. 